\title{
An Information Hiding Algorithm for HEVC Based on Angle Differences of Intra Prediction Mode
}

\author{
Jia-Ji Wang1, Rang-Ding Wang1* ${ }^{*}$, Da-Wen Xu1 ${ }^{1}$, Wei Li ${ }^{1}$ \\ CKC Software Lab, Ningbo University, Ningbo, Zhejiang 315211, China \\ * Corresponding author email: wangrangding@nbu.edu.cn \\ Manuscript submitted January 10, 2014; accepted March 25, 2014.
}

\begin{abstract}
An information hiding algorithm for HEVC is proposed. For 33 kinds of directional prediction modes in the encoding process of $4 \times 4$ luminance blocks, intra prediction modes are mapped to the angle values. We are establishing a mapping relationship table between the angle differences and secret information. Modifying the intra prediction modes to embed secret information is based on the mapping. The extraction of information merely requires decoding the prediction mode from the bit stream. Experimental results show that the proposed hiding algorithm can embed information with little influence on the bit-rate and effectively guarantee the quality of the cover video.
\end{abstract}

Key words: Information hiding, HEVC, intra prediction, angle differences.

\section{Introduction}

In recent years, the digital information is counterfeited, tampered, and attacked, which are increasingly serious phenomenon [1]. Hence, information security problem is increasingly highlighted. Information hiding technology plays an important role in curbing the illegal use of digital multimedia, protecting information from the interference and damage.

HEVC standard is a next-generation video coding standard which is developed by ITU-T Video Coding Experts Group and Moving Picture Experts Group [2]. It is aimed to significantly improve encoding efficiency on the basis of H.264/AVC, especially for dealing with high-definition video. The efficient compression performance and processing capacity of HEVC standard have an important role in high-definition video applications. The information hiding algorithm for HEVC has a theoretical value and practical significance. However, based on HEVC is still in its infancy. At present, the information hiding algorithm based on H.264/AVC has become mature, such as those based on the modified DCT coefficients, motion vector and prediction mode.

Among the information hiding algorithms for H.264/AVC based on the intra prediction mode [3]-[7], Hu et al. [3] modified the intra prediction mode based on the mapping between the secret information and the prediction mode. The mapping relationship is established on the statistical results of multiple video test sequences. Wang et al. [4] embedded secret information in I-frame, P-frame and B-frame through adjustment of the encoding mode of given macroblocks. Xu et al. [5] proposed a hiding information algorithm based on modulating the intra prediction of $4 \times 4$ luminance blocks. If the best mode did not match the information bit, the best prediction mode will be replaced with the substitute mode which is the one with the least Lagrangian cost among those having different parity with the best mode. Yang et al. [6] established a mapping between the information and intra prediction mode with matrix coding. Two bits of secret information is inserted in three intra-frame $4 \times 4$ luminance blocks and only one prediction mode is modified. Yin et al. [7] proposed an algorithm based on Yang's work [6] and utilized an embedding/extracting matrix, three watermark bits are embedded while only one intra 
$4 \times 4$ block's mode is changed.

Combined with the new technology in HEVC intra prediction coding, we have proposed an information hiding algorithm for HEVC based on intra prediction mode and block code [8]. According to the mapping between $(4,3)$ code standard array decoding table and the prediction mode, it makes that three bits of information is embedded in four $4 \times 4$ luminance blocks and modify 1.25 prediction modes on average, which reduce the impact of the prediction mode modulation on the PSNR and bit-rate. The algorithm has a high complexity. This paper presents an information hiding algorithm for HEVC based on angle differences of intra prediction modes. The directional prediction modes are mapped to the angle values. Modifying the intra prediction modes to embed secret information is based on the mapping relationship between the angle differences and secret information. The extraction of information merely requires decoding the prediction mode from the bit stream. The information hiding algorithm is satisfied with the needs of real-time performance.

\section{Intra Prediction in HEVC}

Similar to the H.264/AVC, HEVC adopts the conventional hybrid video coding framework. However, compared to the H.264/AVC, HEVC gives up the concept of macroblock but introduces three basic uints: coding unit(CU), prediction unit(PU)and transform unit(TU).The separation of three basic units can make the procession of prediction, transformation and coding more flexible. The effect after compression can be more in line with the characteristics of video image itself [9].

\subsection{Intra Prediction}

In H.264/AVC standard, intra prediction coding exploits the spatial correlation of pixels. The pixels of the current block are predicted by adjacent pixels in the neighboring blocks that are coded and reconstructed before. The selection process of best intra prediction mode uses the Lagrangian rate-distortion optimization model to choose the minimum rate-distortion cost from all the intra prediction modes [10]. In principle, intra prediction of HEVC adopts the same technology in H.264/AVC, but increases the number of the prediction modes. In Fig.1, there are nine intra prediction modes of $4 \times 4$ luminance block in H.264/AVC. However, HEVC refines more prediction modes and provides as many as 35 intra prediction modes. The increased modes make intra prediction more accurate and reduce the spatial redundancy.
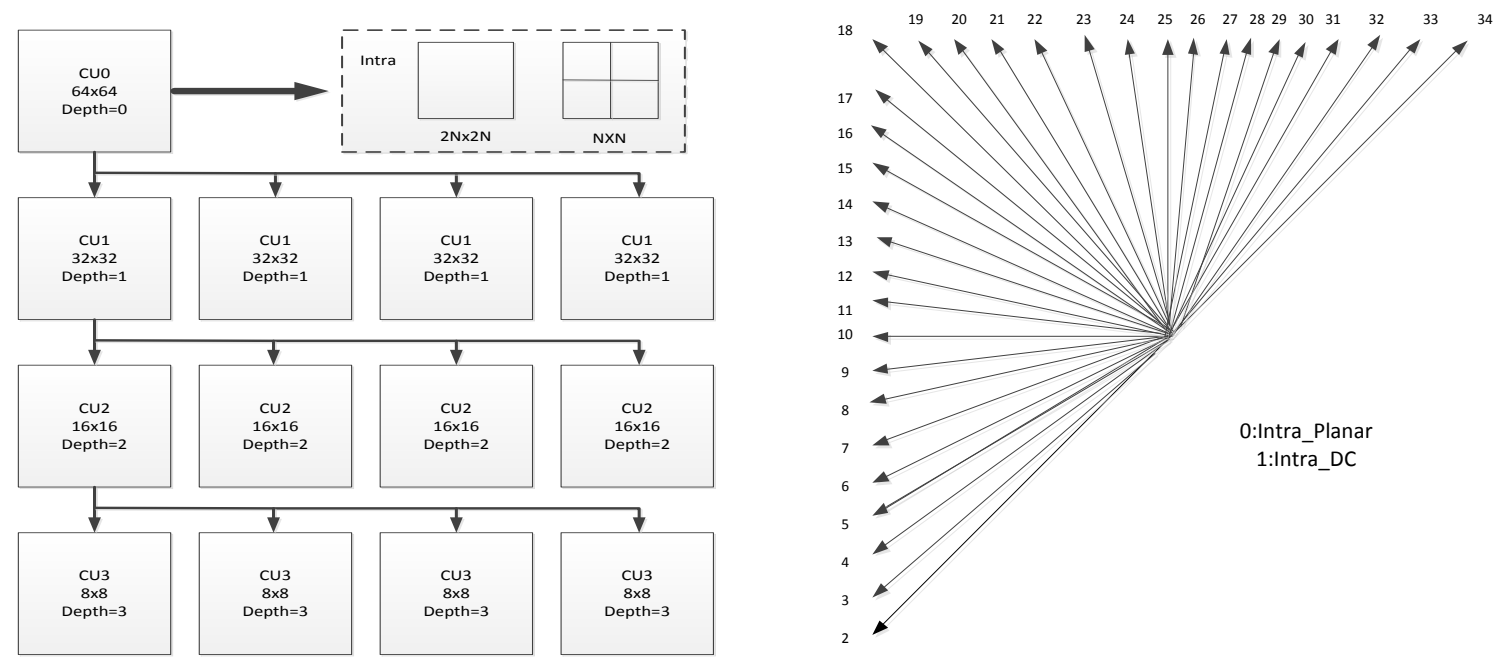

Fig. 1. Intra prediction modes in H.264/AVC and HEVC.

\subsection{The Selection of Intra Prediction Mode in $4 \times 4$ Luminnance Block}

One LCU(Large Coding Unit) quadtree splitting process is described in Fig. 2. Firstly, the splitting process of LCU is conducted. The default size of LCU is $64 \times 64$, and SCU(Small Coding Unit) is $8 \times 8$. When LCU does not split into a smaller size, it becomes CU0 of which size usually is $64 \times 64$ (depth=0). Then it begins to do prediction 
coding of prediction unit as the same size as CU0. At the same time, this CU0 should do all mode selection of different prediction units, finally get the rate-distortion cost J(CU0). Then the CU splits into four smaller sub-cu CU1 and the size of each CU1 is $32 \times 32$. In a similar way, we can get the rate-distortion cost J(CU1) of each CU1. As is shown above, it does a recursive splitting process. When the size of sub-cu is $8 \times 8$, the size of prediction unit can split into four $4 \times 4$ prediction units if it is ready to do prediction coding [11].

So far, the recursive traversal process of LCU is over, LCU begins to do a reverse cutting. When the depth is 3, HEVC needs to compare the sum of cost of four $4 \times 4$ prediction units, namely 4 times J(CU4) with the cost of one $8 \times 8$ prediction unit, namely J(CU3). If J(CU3) is larger, HEVC will choose the $4 \times 4$ coding unit, else it will choose the $8 \times 8$ coding unit. In turn, HEVC can complete the corresponding CU size selection until the depth is 0 .

To select the reasonable and effective optimal mode from the 35 prediction modes, HEVC chooses the optimal prediction mode by using the Lagrangian optimization model. The computation formula is computed by:

$$
J\left(s, c, I M O D E \mid Q P, \lambda_{M O D E}\right)=D(s, c, I M O D E \mid Q P)+\lambda_{M O D E} \cdot R(s, c, I M O D E \mid Q P)
$$

where $Q p$ is the quantization parameter and $\lambda_{M O D E}$ is the Lagrange multiplier. $s$ and $c$ represent the original block and reconstruction block respectively. $R$ is the bit-rate and $D$ is the distortion degree.

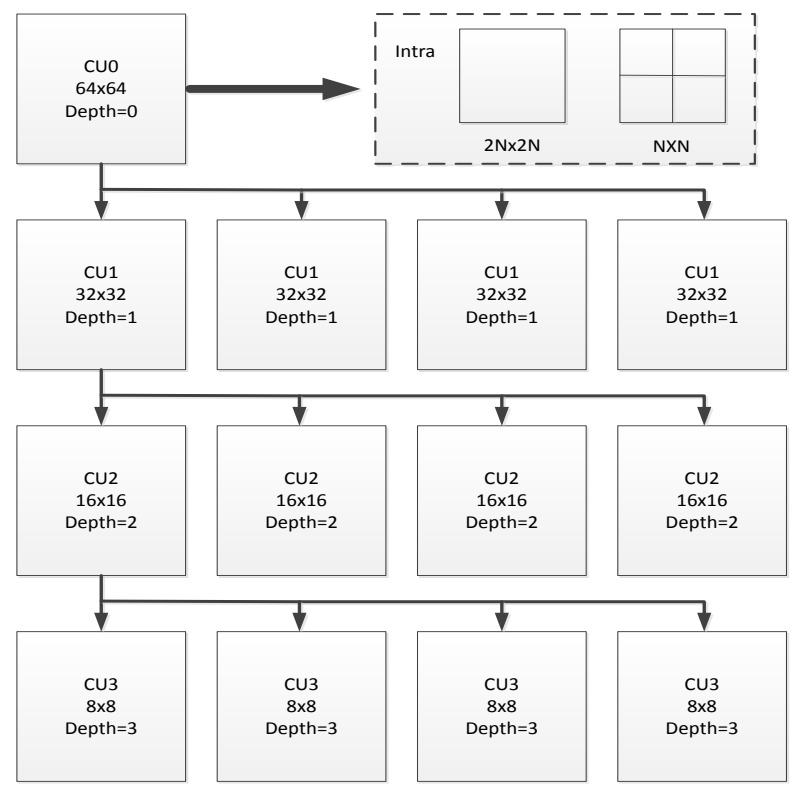

Fig. 2. The process of quadtree partitioning.

\section{Information Hiding Algorithm}

In this paper, the intra prediction modes in $4 \times 4$ luminance blocks are modified to embed secret information. According to the mapping relationship between the secret information and angle differences of prediction mode, the corresponding prediction mode is modified to embed the secret information.

\subsection{The Principle of Information Hiding}

For the traditional LSB (low significant bit) embedding technique applied in information hiding, secret information and LSB are evenly distributing under prevailing conditions. If the information is embedded in all LSB, half of LSB will be changed. In order to improve the quality of host carrier with secret information, the number of the modified bits in host carrier should be reduced as much as possible. This paper presents an information hiding algorithm based on angle differences of intra prediction modes. In the intra encoding process, HEVC provided 35 kinds of prediction modes, including 33 kinds of directional modes. The angle difference between two neighboring modes is $\pi / 32$. We establish the mapping between the prediction mode and the angle 
values as shown in Table 1. Because the prediction method of mode 0 and mode 1 is independent of direction, two prediction modes are not mapped into the angle values. The angle difference of two prediction modes is embedded two bits of secret information. The two prediction modes simply modify 0.75 mode on average, which reduce the modified bits to ensure that the objective and subjective quality of the video.

Table 1. The Mapping Table between the Prediction Mode and Angle

\begin{tabular}{cccccc}
\hline \hline Prediction Mode & Angel & Prediction Mode & Angle & Prediction Mode & Angle \\
\hline 0 & N/A & 12 & $-\pi / 16$ & 24 & $-7 \pi / 16$ \\
1 & N/A & 13 & $-3 \pi / 32$ & 25 & $-15 \pi / 32$ \\
2 & $\pi / 4$ & 14 & $-\pi / 8$ & 26 & $-\pi / 2$ \\
3 & $7 \pi / 32$ & 15 & $-5 \pi / 32$ & 27 & $-17 \pi / 32$ \\
4 & $3 \pi / 16$ & 16 & $-3 \pi / 16$ & 28 & $-9 \pi / 32$ \\
5 & $5 \pi / 32$ & 17 & $-7 \pi / 32$ & 29 & $-19 \pi / 32$ \\
6 & $\pi / 8$ & 18 & $-\pi / 4$ & 30 & $-5 \pi / 8$ \\
7 & $3 \pi / 32$ & 19 & $-9 \pi / 32$ & 31 & $-21 \pi / 32$ \\
8 & $\pi / 16$ & 20 & $-5 \pi / 16$ & 32 & $-11 \pi / 16$ \\
9 & $\pi / 32$ & 21 & $-11 \pi / 32$ & 33 & $-23 \pi / 32$ \\
10 & 0 & 22 & $-3 \pi / 8$ & 34 & $-3 \pi / 4$ \\
11 & $-\pi / 32$ & 23 & $-13 \pi / 32$ & & \\
\hline \hline
\end{tabular}

For two consecutive $4 \times 4$ luminance blocks, the optimal prediction modes are extracted. If two prediction modes are within $[2,34]$, the modes are mapped into the angle values which is denoted by $D_{1}$ and $D_{2}$. We calculate the absolute value of the angle difference between $\mathrm{D}_{1}$ and $\mathrm{D}_{2 .}$. According to the mapping relationship between two bits of secret information and angle difference as shown in Table 2, we modify the angle difference to embed the secret information. Modifying the prediction mode will affect the quality of the video. If the difference between the optimal prediction mode and the substitutive prediction mode is obvious, it will have a great influence on the subjective and objective quality of video. Here the secret information corresponding to the angle difference ranges are scattered. When the prediction mode is modified to embed information, we hope to change the optimal prediction mode to the substitutive prediction mode which is close to the original video effect.

We do not modify the prediction mode if the value of angle difference $\mathrm{D}$ in the interval of difference which is corresponding to the two bits secret information. Or we modify the prediction mode of the second luminance block to make the angle difference $\mathrm{D}^{\prime}$ in the interval. For the modifying prediction mode of the second luminance block, we search the angle value which meet the requirements and map it into prediction mode according to the Table 1. Then we calculate the rate distortion cost of luminance block to choose the corresponding prediction mode which has minimum rate distortion cost as the current intra $4 \times 4$ luminance block. The current block is recoded with the prediction mode above.

If two prediction modes of the $4 \times 4$ luminance blocks are 0 or 1 , the mode can't be mapped into the angles because the prediction method of mode 0 and mode 1 is independent of direction. In order to ensure the capacity of secret information embedding, we modify the mode 0 and mode 1 to complete the embedding based on the secret information. Fig. 3 presents the probability distribution of the sub-optimal prediction mode when the optimal mode is determined. As can be seen from the figure, the sub-optimal mode would be mode 1 (mode 0 ) with a high probability when the optimal mode is equal to 0 (or 1 ). If the prediction mode changes from 0 to 1 or from 1 into 0 , it will not cause a significant impact on the quality of the cover video. For each $4 \times 4$ luminance block, we modify the prediction mode to embed one bit secret information. If the secret information is 0 , then the prediction mode is changed into 0 . If the secret information is 1 , then the prediction mode is changed into 1 . If one of prediction mode in two $4 \times 4$ luminance blocks is 0 or 1 , and another mode is within $[2,34]$, we don't embed secret information. 
Table 2. The Mapping Table between the Prediction Mode and Angle Range

\begin{tabular}{cccc}
\hline \hline Angle Range & Secret & Angle Range & Secret \\
\hline $0 \sim \pi / 16$ & Information & $\pi / 2 \sim 9 \pi / 16$ & Information \\
$\pi / 16 \sim \pi / 8$ & 00 & $9 \pi / 16 \sim 5 \pi / 8$ & 00 \\
$\pi / 8 \sim 3 \pi / 16$ & 01 & $5 \pi / 8 \sim 11 \pi / 16$ & 10 \\
$3 \pi / 16 \sim \pi / 4$ & 10 & $11 \pi / 16 \sim 3 \pi / 4$ & 11 \\
$\pi / 4 \sim 5 \pi / 16$ & 11 & $3 \pi / 4 \sim 13 \pi / 16$ & 00 \\
$5 \pi / 16 \sim 3 \pi / 8$ & 00 & $13 \pi / 16 \sim 7 \pi / 8$ & 01 \\
$3 \pi / 8 \sim 7 \pi / 16$ & 01 & $7 \pi / 8 \sim 15 \pi / 16$ & 10 \\
$7 \pi / 16 \sim \pi / 2$ & 10 & $15 \pi / 16 \sim \pi$ & 11 \\
\hline \hline
\end{tabular}
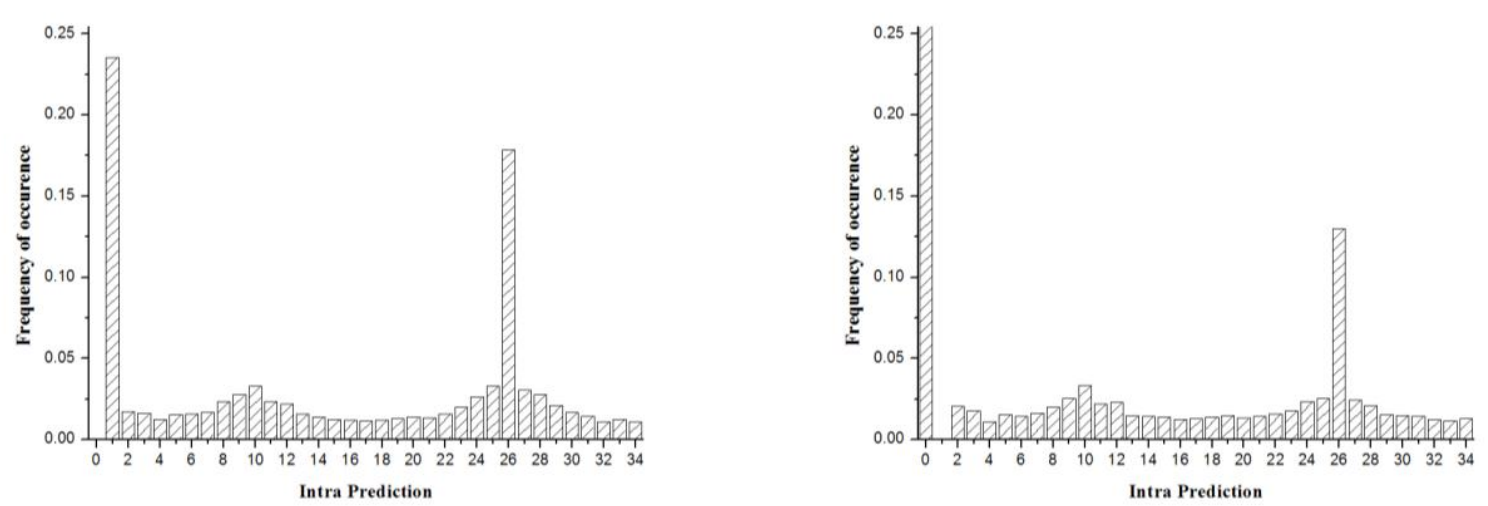

Fig. 3. The probability distribution of the sub-optimal prediction mode.

\subsection{Data Embedding Procedure}

In the proposed method, we embed the secret information according to the mapping between the angle difference and the secret information. The hiding algorithm is described as following:

Step1. For two consecutive $4 \times 4$ luminance blocks, we extract the optimal prediction modes.

Step2. The prediction modes are mapped into the angles. According to the mapping relationship between the secret information and angle differences of prediction modes, the corresponding prediction mode is modified to embed the secret information. Re-encode the $4 \times 4$ luminance block with the substitutive prediction mode.

Step3. Compare the $4 \times 4$ blocks with other encoding modes with the Lagrangian cost. If the cost of the $4 \times 4$ blocks is less than the other encoding modes, save the embedded information. Otherwise, embed the current bits next time without saving the embedded bits.

Step4. Repeat the above steps until embedding all the secret information.

\subsection{Data Detection Procedure}

Secret data detection procedure is simple, only need to decode part of I frame. The steps are described as following:

Step1. Determine whether the block coding mode of current block is $4 \times 4$ type. If the current block coding mode is other coding mode, go to Step3 without extracting the secret information. If the block coding mode is $4 \times 4$ type, decode the prediction modes of two consecutive $4 \times 4$ luminance blocks including current block.

Step2. If two prediction modes are within [2,34], the modes are mapped into the angle values and calculate the absolute value of the angle difference. We extract the secret information according to the mapping between the angle difference and the secret information.

Step3. Repeat the Step1 and Step2 for the $4 \times 4$ blocks until extracting all the information.

\section{Experimental Results and Discussions}


This algorithm has been simulated in the HM-12.0 model of the HEVC reference software. We select five different sequences (Vidyo_1, Vidyo_3, Vidyo_4, BasketballDrill and BQMall) for testing. The main reference software coding configuration parameters are shown in Table 3, and the remaining parameters are set to the default configuration.

\begin{tabular}{lc} 
Table 3. Configuration Parameters of the HM \\
\hline \hline Parameters & Configuration \\
\hline Frames To Be Encoded & 96 \\
Frame Rate & $30 \mathrm{fps}$ \\
Intra Period & 16 \\
GOPSize & 8 \\
RDOQTS & 1 \\
\hline
\end{tabular}

Fig. 4 shows the reconstruction of video image before and after embedded secret information. The original reconstructed frame is shown as Fig. 4(a). The marked reconstructed frame with the hidden information is shown as Fig. 4(b). As shown in the picture, information hiding will not have any noticeable impact on perceptual video quality.

In addition to judge the subjective quality of the video, experiments also evaluate algorithm from the objective video coding quality variation (PSNR), bit rate variation (BRI) and data hiding capacity (HBQ). BRI and PSNR are defined as follows:

$$
\begin{gathered}
B R I=\frac{R^{\prime}-R}{R} \times 100(\%) \\
P S N R=P S N R_{Y}^{\prime}-P S N R_{Y}
\end{gathered}
$$

where $R$ and $R^{\prime}$ are the bit-rate before and after embedded secret information, $P S N R_{Y}^{\prime}$ and $P S N R_{Y}$ are the video coding quality before and after embedded secret information.

Fig. 5 shows the PSNR curves of the original and hidden information video. As can be seen from the diagram, $P S N R$ value is slightly lower after embedding the secret information, and the PSNR difference between original and embedded video is within $0.06 \mathrm{~dB}$.

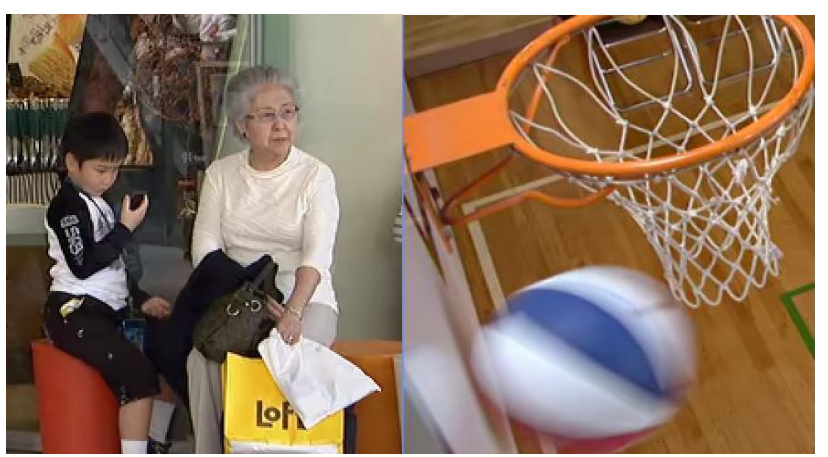

(a) Original reconstructed frame.

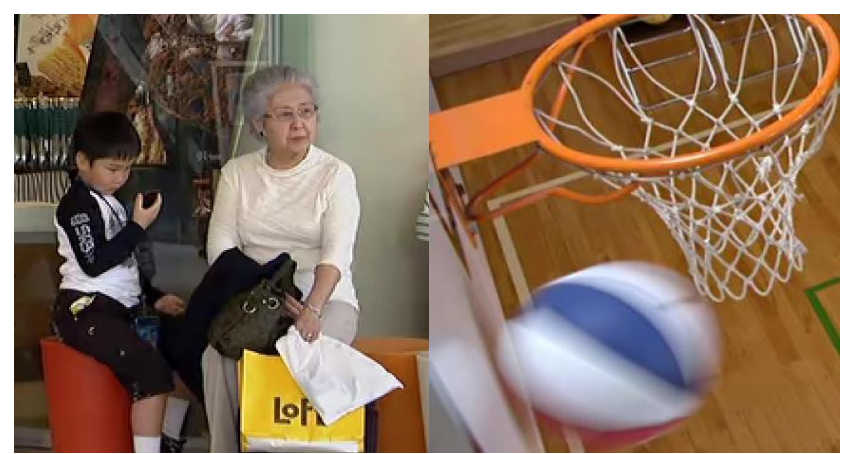

(b) Marked reconstructed frame.

Fig. 4. Comparison of the visual quality between the original and the marked frames.

Table 4 lists the experimental results of the comprehensive performance of the test sequence. For the different video test sequences, the number of $4 \times 4$ luminance blocks leads to the difference of embedding capacity. For the same video test sequence, the embedding has little effect on the video quality and meet the visual concealment. Since we only modified the optimal prediction mode into the substitutive prediction mode which has similar direction and matches condition, the residuals is small. And we re-encode the $4 \times 4$ luminance block with the substitutive prediction mode. It can effectively guarantee the quality of the cover video. 


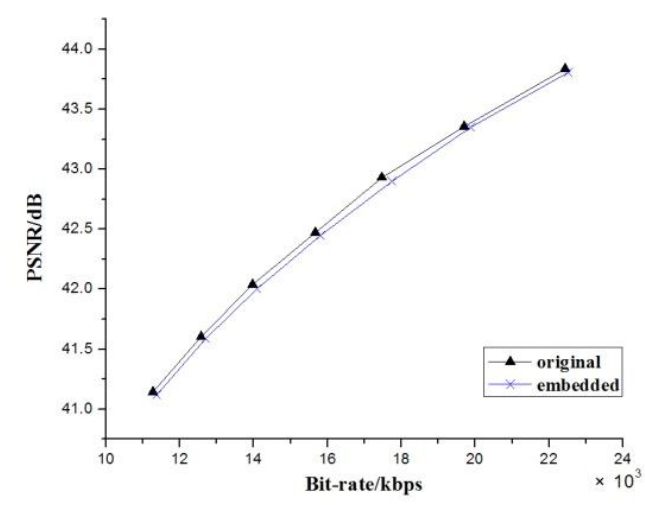

(a) Vidyo_1

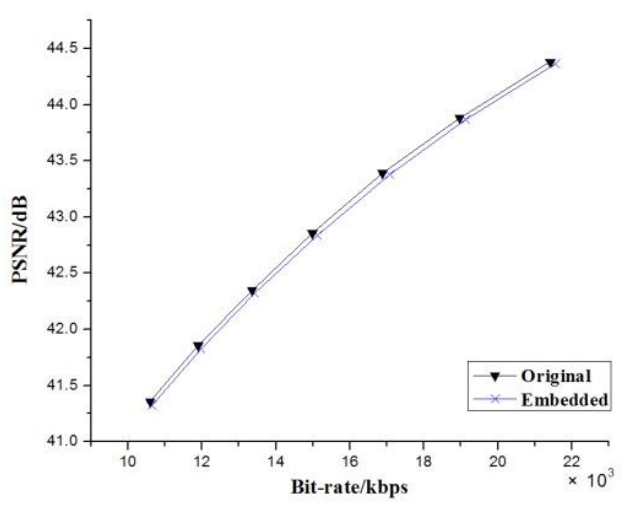

(c) Vidyo_4

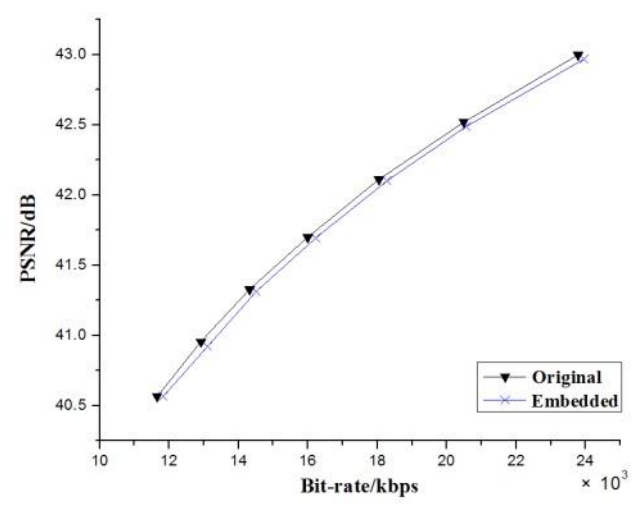

(b) Vidyo_3

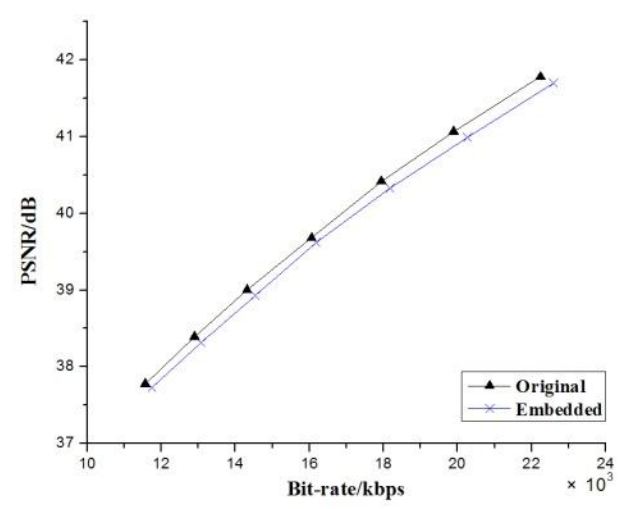

(d) Basketball Drill

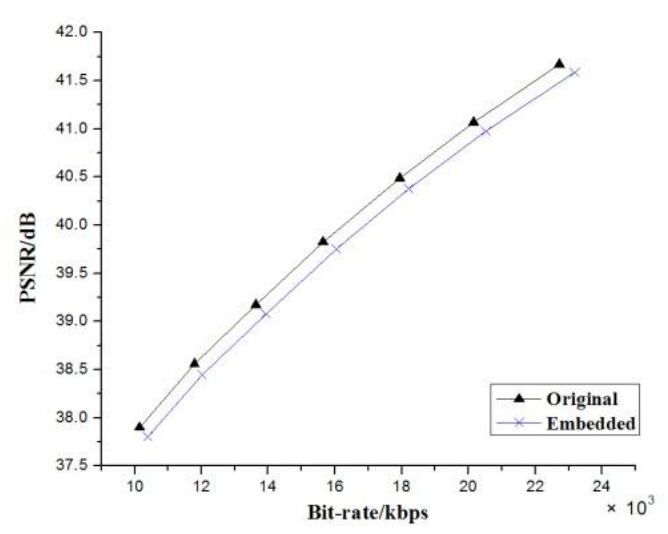

(e) BQMall.

Fig. 5. The variation of PSNR induced by data hiding.

Table 4. Experimental Results of the Comprehensive Performance

\begin{tabular}{lccc}
\hline \hline Sequence & BRI(\%) & $\Delta$ PSNR & HBQ(bits) \\
\hline BasketballDrill & 1.90 & -0.06 & 11070 \\
Vidyo1 & 0.76 & -0.04 & 9534 \\
Vidyo3 & 0.45 & -0.01 & 8058 \\
Vidyo4 & 0.46 & -0.03 & 5502 \\
BQMall & 1.58 & -0.06 & 16182 \\
\hline \hline
\end{tabular}


Compared with the different bit streams, the value of PSNR may be not to reflect the relative quality of the different video. As a result, we introduce an estimate index named SSIM which is an index to measure the similarity of two images [12]. The value of SSIM falls in between 0 and 1 . The value is greater, the similarity is higher. As we can see from the Fig. 6, the values of SSIM in all sequences are around 0.97, and the embedded information has little effect on the quality of the video.

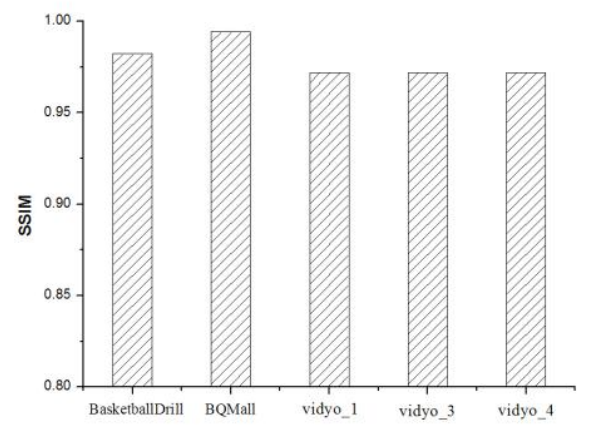

Fig. 6. Experimental results for video quality (SSIM).

In addition to the experimental analysis for embedding secret information on video quality impacts, we analyze the safety of the proposed algorithm. The secret information can be extracted intact in the absence of any attack. In order to detect the robust of the algorithm, we do the recompression test with the same quantization parameter to recompress the video test sequences which are embedded secret information. Through the testing we find that the embedded secret information can't be properly extracted after the recompression. What's more, the error rate is about $40 \%$. It means that if the video bit stream with the embedded secret information is attacked, the secret information can't be extracted intact. The main factor is that the prediction modes change a lot after the compression, which is influenced by the variation of luminance pixels. In conclusion, the proposed algorithm is fragile.

\section{Conclusion}

In this paper, an information hiding algorithm for HEVC is proposed. The directional prediction modes are mapped to the angle values. According to the mapping relationship between the secret information and angle differences of prediction mode, the corresponding prediction mode is modified to embed the secret information. Experiments show that the algorithm extraction process is simple, rapid and little impact on the video after embedding information.

\section{Acknowledgment}

This work is sponsored by K.C. Wong Magna Fund in Ningbo University, National Natural Science Foundation of China (NSFC: 61170137, 61300055, 61301247), Doctoral Fund of Ministry of Education of China(20103305110002), Zhejiang natural science foundation of China (ZJNSF: LY13F020013), Ningbo natural science foundation of China (2013A610057, 2013A610059), Scientific Research Fund of Zhejiang Provincial Education Department (Y201119434), Open Fund of Zhejiang Provincial Top key Discipline of Information and Communication Engineering (XKXL1313, XKXL1310).

\section{References}

[1] Cox, I. J., Miller, M. L., \& Bloom, J. A. (2002). Digital Watermarking. USA: Elsevier Science.

[2] Han, G. J., Ohm, J. R., Han, W. J., et al. (2012). Overview of the high efficiency video coding (HEVC) standard. IEEE Trans. on, Circuits and Systems for Video Technology, 22(12), 1649-1668.

[3] Hu, Y., Zhang, C., \& Su, Y. (2008). Information hiding for H.264/AVC. Acta Electronica Sinica, 36(4), 690-694. 
[4] Wang, R., Zhu, H., \& Xu, D. (2010). Information hiding algorithm for H.264/AVC based on encoding mode. Opto-Electronic Engineering, 37(5), 144-150.

[5] Xu, D. W., Wang, R. D., \& Wang, J. C. (2014). Prediction mode modulated data-hiding algorithm for H.264/AVC. Journal of Real-Time Image Processing, 7(4), 205-214.

[6] Yang, G. B., Li, J. J., He, Y. L., \& Kang, Z. W. (2011). An information hiding algorithm based on intra-prediction modes and matrix coding for H.264/AVC video stream. AEU-International Journal of Electronics and Communications, 65(4), 331-337.

[7] Yin, Q., Wang, H., \& Zhao, Y. (2012). An information hiding algorithm based on intra-prediction modes for H.264 video stream. Journal of Optoelectronics Laser, 23(11), 2194-2199.

[8] Wang, J., R., Wang, W., Li, Xu, D., \& Huang. M. (2014). An Information hiding algorithm for HEVC based on intra prediction mode and block code. Sensors \& Transducers, 177(8), 230-237.

[9] Ohm J., Sullivan, G. J., Schwarz, H., et al. (2012). Comparison of the coding efficiency of video coding standards-including high efficiency video coding (HEVC). IEEE Trans. on Circuits and Systems for Video Technology, 22(12), 1669-1684.

[10] Lainema, J., Bossen, F., Han, W. J., et al. (2012). Intra coding of the HEVC standard. IEEE Trans. on Circuits and Systems for Video Technology, 22(12), 1792-1801.

[11] Kim, I. K., Min, J., Lee, T., et al. (2012). Block partitioning structure in the HEVC standard. IEEE Trans. on Circuits and Systems for Video Technology, 22(12), 1697-1706.

[12] Wang, Z., Bovik, A. C., Sheikh, H. R., \& Simoncelli, E. P. (2004). Image quality assessment: from error visibility to structural similarity. IEEE Trans. on, Image Processing, 13(4), 600-612.

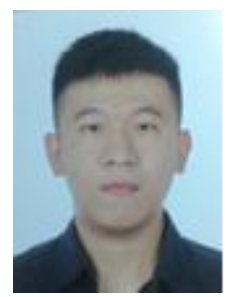

Jia-Ji Wang received the B.S. degree in communication engineering from Heilongjiang University. He is currently working toward the master degree at Ningbo University. His research interests information hiding.

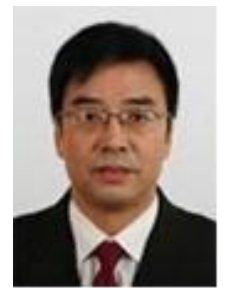

Rang-Ding Wang received the M.S. degree from Northwest Polytechnic University, China, in 1987, and the Ph.D. degree from TongJi University, China, in 2004. He is a Professor at Faculty of Information Science and Engineering, Ningbo University, China. His research interests include multimedia information security, digital speech processing, digital watermarking, steganography and steganalysis. He is an author/coauthor of more than 120 research papers, and two books. He holds more than 20 patents.

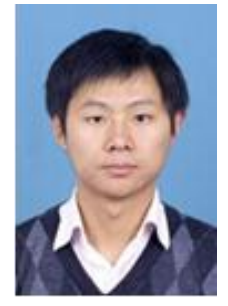

Da-Wen Xu received the M.S. degree in communication and information system from Ningbo University, China, in 2005 and the Ph.D degree in computer applied technology from Tongji University, China, in 2011. He is an associate professor at School of Electronics and Information Engineering, Ningbo University of Technology, China. His research interests mainly include digital watermarking and information hiding, signal processing in the encrypted domain. He has served as a technical paper reviewer for IEEE conferences, journals \& magazines.

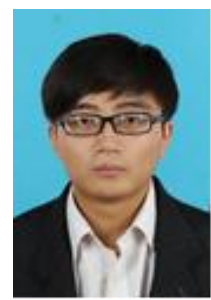

Wei Li received the B.S. degree in communication engineering from Shandong Agricultural University. He is currently working toward the master degree at Ningbo University. His research is multimedia communications. 